\title{
Descriptive Seasonal Adjustment by \\ Minimizing Perturbations
}

\author{
by \\ EKKEHART SCHLICHT \\ and \\ RALF PAULY
}

Empirica

1983

number 1

pages $15-28$

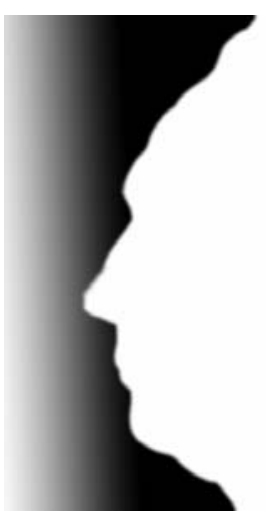

published by

www.semverteilung.vwl.uni-muenchen.de 


\title{
Descriptive Seasonal Adjustment by Minimizing Perturbations ${ }^{\star}$ )
}

\author{
Ekkehart Schlicht, Ralf Pauly
}

\section{Zusammenfassung}

Das von Schlicht (1981) vorgeschlagene Saisonbereinigungsverfahren kann als ein Verfahren aufgefaßt werden, welches Perturbationen (nichtstochastische Störungen) minimiert. Diese Sichtweise führt zu einer Kritik des dort vorgeschlagenen Saisonkriterismus. Es wird ein neues Saisonkriterium vorgeschlagen, welches diese Nachteile nicht aufweist, und es wird das resultierende Saisonbereinigungsverfahren angegeben.

\section{Introduction}

The descriptive decomposition of a given time series into trend, seasonal component, and irregular component can be formulated as an optimization problem (Schlicht, 1981). The trend is to be chosen such that it is as smooth as possible; the seasonal component is to be chosen such that it exhibits a seasonal pattern as stable as possible; and trend and seasonal components ought to approximate the given time series as well as possible, $i . e$. the irregular component is to be minimized.

If $x^{\prime}=\left(x_{1}, \ldots, x_{T}\right)$ denotes the given time series of length $T, y^{\prime}=\left(y_{1}, \ldots, y_{T}\right)$ is the trend, $z^{\prime}=\left(z_{1}, \ldots, z_{T}\right)$ denotes the seasonal component and $u^{\prime}=\left(u_{1}, \ldots, u_{T}\right)=x^{\prime}-y^{\prime}$ $-z^{\prime}$ denotes the irregular component, it is necessary to specify a trend criterion $f(y)$ : $\mathrm{R}^{T} \rightarrow \mathrm{R}$ measuring the curvature of the trend, a seasonality criterion $\mathrm{g}(z): \mathrm{R}^{T} \rightarrow \mathrm{R}$ measuring the instability of the seasonal component, and an irregularity criterion $h(u): R^{T} \rightarrow R$ measuring the size of the irregular component. If

$$
\text { (1) } f(y)+g(z)+h(x-y-z)=\underset{y . z}{\min !}
$$

is minimized with respect to trend $y$ and seasonal component $z$, this determines a trend and a seasonal component which minimize jointly the sum of the three criteria: curvature of the trend, instability of the seasonal component, and size of the irregular component.

The purpose of this paper is to modify the seasonality criterion proposed in Schlicht (1981) while maintaining the trend and irregularity criteria and to prove that the modified set of criteria leads again to a unique solution of the decomposition problem without having recourse to initial values.

") We thank P. A. Cholette and H. Lütkepohl and an anonymous referee for helpful hints. 
The view adopted is that the sum of the criteria (1) can be interpreted as a weighted sum of squares of individual perturbations - as a weighted regression. These perturbations are viewed here as exogenous shocks and not as random disturbances governed by a statistical law(1). This view is slightly different from the view adopted in Schlicht (1981), but if it is adopted, it will be argued, this requires a reformulation of the seasonality criterion.

The paper is organized as follows: In section 1, the criteria proposed in Schlicht (1981) are reformulated in terms of perturbations. This leads to criticism of the seasonality criterion (section 2). In section 3, a new seasonality criterion is introduced and compared with the older criterion. In section 4 it will be proved that a unique decomposition can be obtained if the old seasonality criterion is replaced by the new one. Some concluding remarks follow in section 5 .

\section{The criteria as measures of perturbations}

The criteria proposed in Schlicht (1981) can be written as quadratic forms, namely:

(2) $f(y)=\alpha y^{\prime} P^{\prime} P y \quad \alpha>0$

(3) $g(z)=\gamma z^{\prime} S^{\prime} S z \quad \gamma>0$

(4) $\dot{h}(u)=u^{\prime} u$

where

(5)

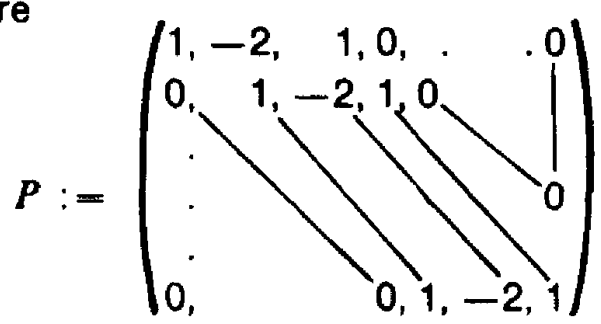

of order $(T-2) \times T$,

(6) $S$ from $S^{\prime} S=\left(R^{\prime} R+\frac{\beta}{\gamma} \cdot Q^{\prime} Q\right) \quad \beta \geq 0$

with

column $s$

$\downarrow$

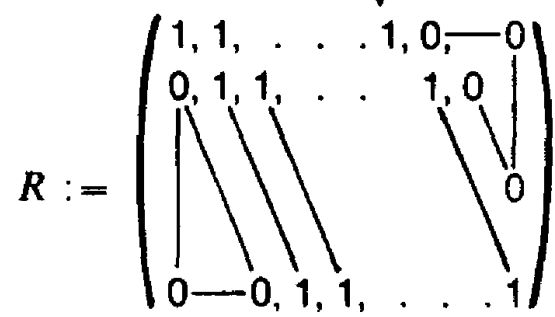




$$
\text { of order }(T-s+1) \times T \text {, and }
$$

(8)

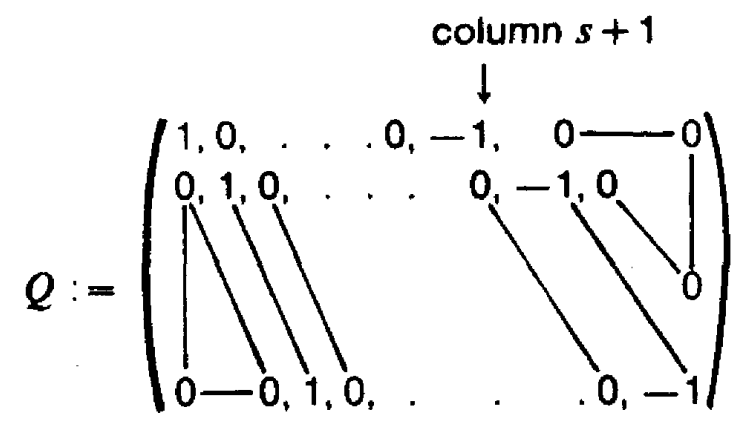

of $\operatorname{order}(T-s) \times T$

where $s$ denotes the length of the season (i. e. $s=12$ in the case of monthly data).

The "perturbations" interpretation of the trend and irregularity criteria is straightforward:

The trend perturbations $v^{\prime}=\left(\nu_{3}, \ldots, v_{T}\right)$ represent changes in the direction of the trend, i. e.

(9)

$$
v_{1}=\left(y_{1}-y_{t-1}\right)-\left(y_{t-1}-y_{t-2}\right) \quad t=3, \ldots, T
$$

If the perturbations are viewed as causes, this amounts to

$$
y_{t}=2 y_{t-1}-y_{t-2}+v_{t} \quad t=3, \ldots, T .
$$

The trend criterion (2) is simply the sum of squared perturbations

(11) $f(y)=\alpha v^{\prime} v$.

Keeping the trend criterion as small as possible means keeping the perturbations in the direction of the trend as small as possible and hence the trend as smooth as achievable.

The irregularity criterion (4) can be interpreted directly as the sum of squares of perturbations $u^{\prime}=\left(u_{1}, \ldots, u_{T}\right)$ where

(12)

$$
u_{t}=x_{t}-y_{t}-z_{t} \quad t=1, \ldots T
$$

or 
Minimizing the irregularity criterion amounts to keeping the irregular perturbations - which are the differences between the original time series and the sum of trend and seasonal components - as small as feasible.

We shall maintain this in the following.

The "perturbations" interpretation of the seasonality criterion is more difficult, however. First, there are several alternatives: We can choose $S$ of order $T \times T$, but then no intuitive interpretation seems to be available. Another way is to take:

(14) $S=\left(\frac{R}{\delta Q}\right)$

where $\delta=\left(\frac{\beta}{\gamma}\right)^{1 / 2}$

This has a direct interpretation in terms of the perturbations

$$
r=R z
$$

and

(16) $q=\delta Q z$

The perturbation

(17) $r_{t}=\sum_{t=0}^{s-1} z_{t-t} \quad t=s, s+1, \ldots, T$

measures the deviation of the seasonal pattern from an average of zero and the perturbation

(18) $q_{t}=-\delta\left(z_{t}-z_{t-s}\right)$

measures the change of the seasonal components from one year to the next. However, $r$ and $q$ cannot be taken to be (functionally) independent since they are directly connected by the seasonal component. Hence the minimization of their sum of squares - as implied by (1) and (3) - makes not very much sense. This difficulty is illuminated by reformulating (1) as a weighted regression:

(19) minimize $u^{\prime} u+\alpha v^{\prime} v+\gamma r^{\prime} r+\gamma q^{\prime} q$ with respect to $y$ and $z$ subject to 


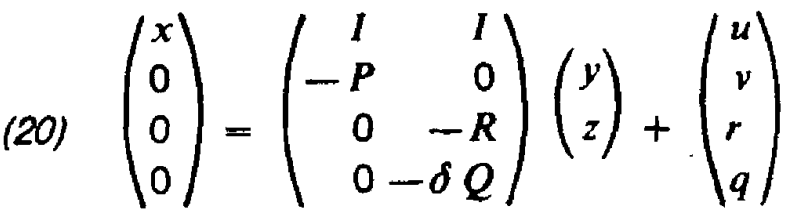

Here the disturbances $r$ and $q$ measure closely related deviations separately(2). Furthermore, the interpretation and choice of $\beta$ raises some problems.

Hence some reflections on the seasonality criterion are needed.

\section{A criticism of the two-part seasonality criterion}

In order to study the change of the seasonal pattern implied by (14), we trace the system's response to the so-called "typical shock" in one period (Sims, 1980): We look at the time profile of the impact of a perturbation injected at time $t$ while keeping all other perturbations hypothetically equal to zero.

Under the specification (14), the seasonality criterion (3) can be written in two-part form (compare also (19)):

$$
\begin{aligned}
g(z) & =\gamma r^{\prime} r+\gamma q^{\prime} q \\
& =g_{1}(z)+g_{2}(z)
\end{aligned}
$$

First we shall look at the impact of one perturbation $r_{1}$ on $g_{1}$ while keeping the other perturbations $r_{\tau}, \tau \neq t$ equal to zero. By evaluating this from (17), we arrive at figure 1 .

It turns out that $r_{t}$ increases $z_{t}$ by $r_{t}$ and decreases $z_{t+1}, z_{t+s+1}, z_{t+2} s+1, \ldots$ by the same amount, leaving all other components unaffected. This is not an attractive feature since it is reasonable to assume the effect of a perturbation $r_{t}$ on other seasonal components $z_{t}$, $\tau>t$ to be more evenly distributed.

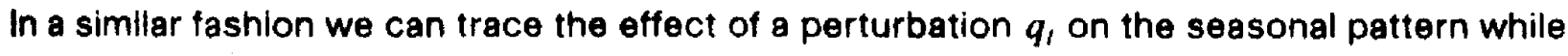
keeping all $q_{\tau}, \tau \neq t$ equal to zero by evaluating (18) (figure 2).

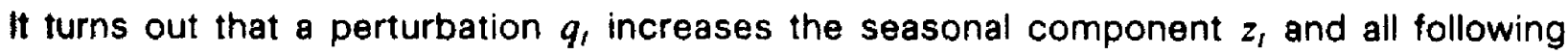
$z_{1+n s}$ referring to the same period in the seasonal pattern without affecting the other seasonal components. This is not a convincing mechanism either because it increases the sum of the seasonal components permanently. Such a permanent increase, however, is to be reckoned as an increase in the trend level. The effect of several perturbations can be 
The impact of a perturbation in the firet pert of the seasonallty criterion on the seasonal pattem

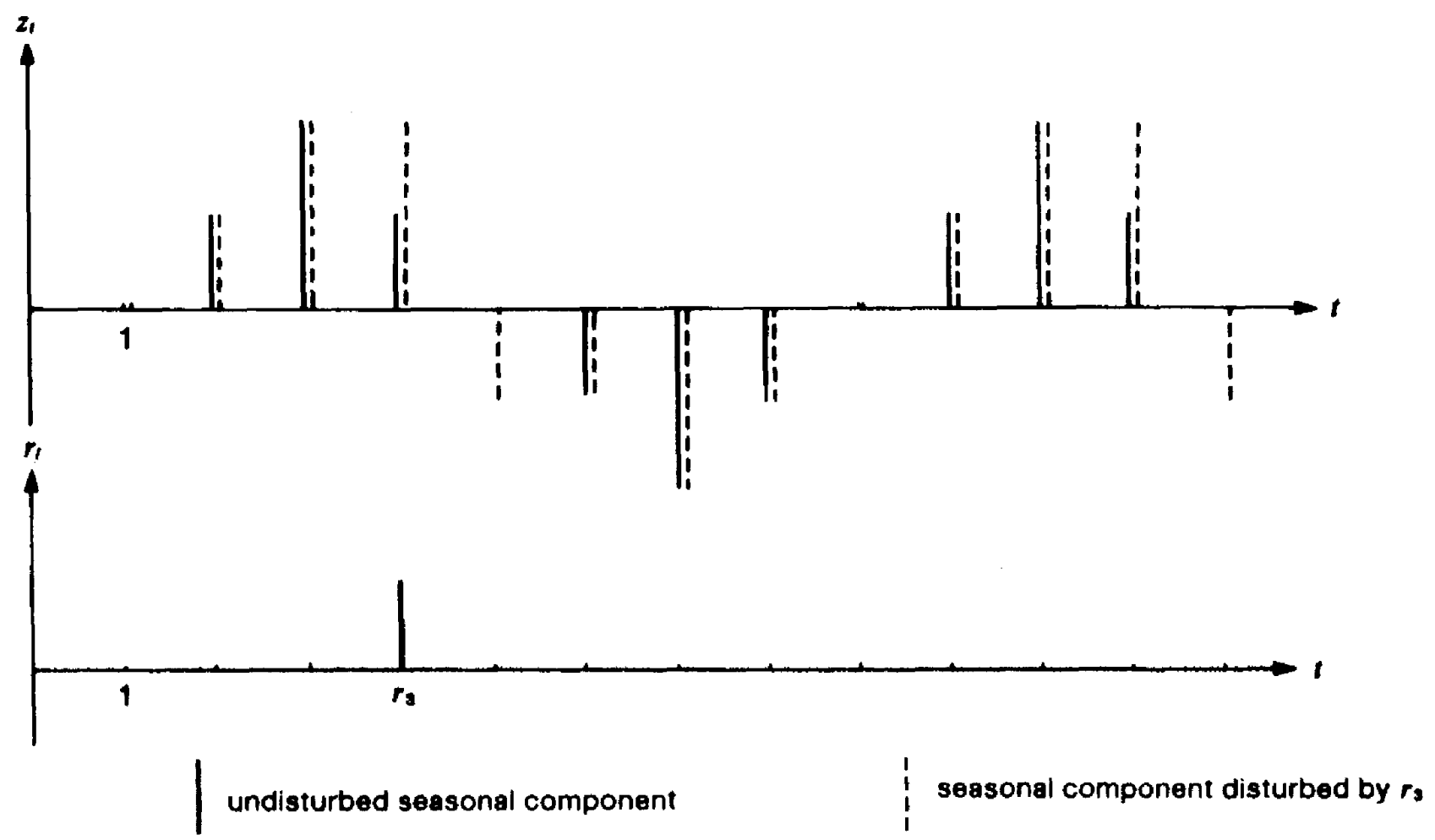

thought of as the summation of the effects of individual perturbations. Hence the problems illustrated above will remain.

If these two aspects are combined in the two-part seasonality criterion - or in the weighted regression (19), (20) - this will mitigate both disadvantages, but it will not necessarily be a reasonable compromise.

\section{A new seasonallty criterion}

If we want to keep the sum of the seasonal components close to zero, this will necessarily induce a negative impact on the following seasonal components. Under the criterion $g_{1}$, this is accomplished by simply reducing the following seasonal component by the increase in the current seasonal component as can be seen from (17) if we re-write it as

(22) $z_{t}=z_{t-s}+r_{t}-r_{t-1}$.

A more sensible formulation would be to distribute the negative impact of a perturbation evenly over the whole length of the season. This leads to 


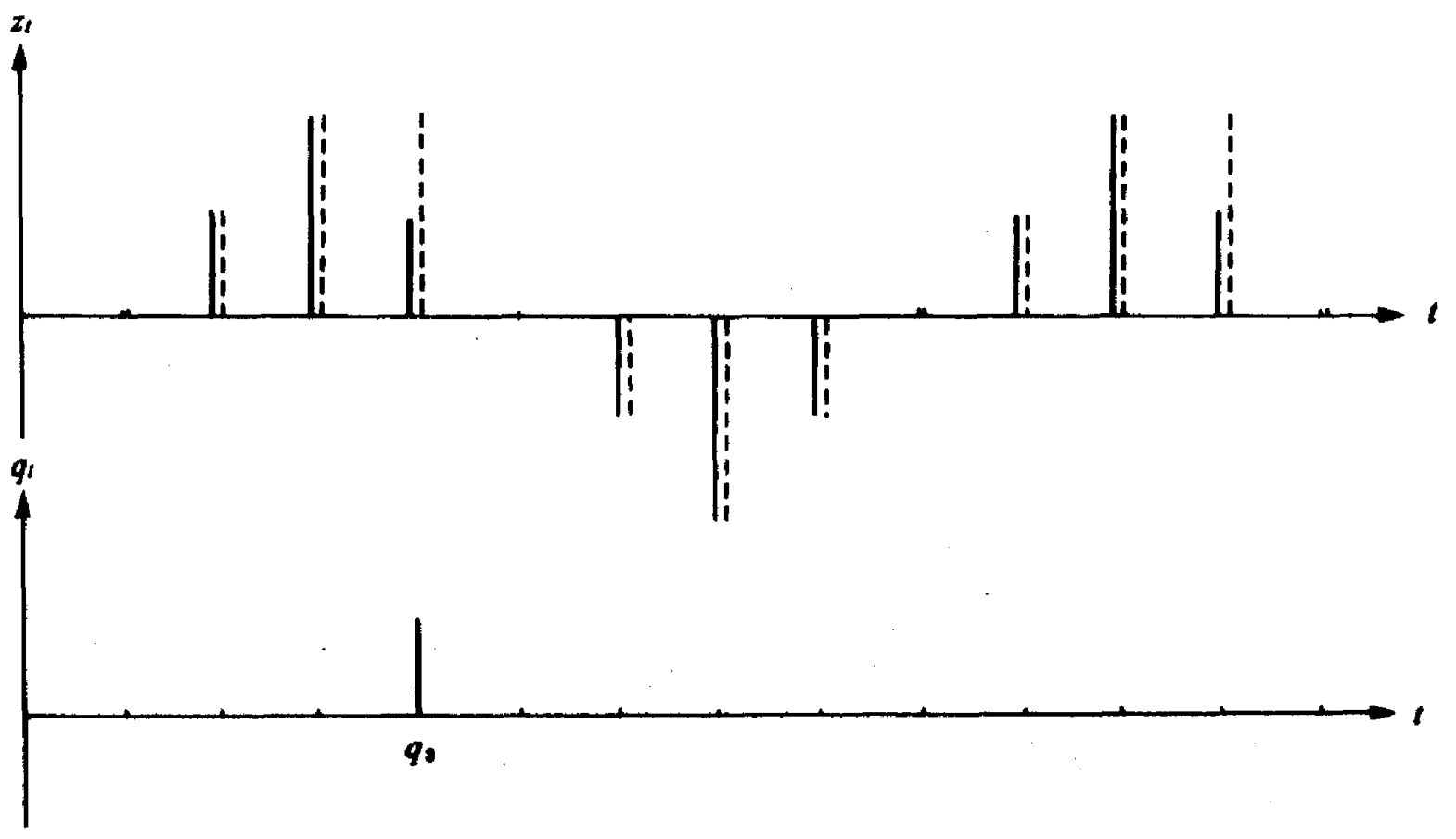
undisturbed seasonal pattern seasonal pattern disturbed by $q_{3}$

(23) $z_{t}=z_{t-s}+w_{t}-\frac{1}{s-1} \sum_{\tau=1}^{s-1} w_{t-\tau} \quad t=s+1, \ldots, T$

where $w^{\prime}=\left(w_{2}, \ldots, w_{T}\right)$ is the perturbation in the new seasonality criterion to be developed(3). The impact of a single perturbation is illustrated in figure 3.

The perturbation $w_{t}$ can be thought of as acting quite similarly as the perturbation $q_{t}$. The difference is simply that the increase in the sum of the seasonal components induced by an increase in one single component is eliminated - as should be done; and, in fact, the similarity between (18) and (23) is quite obvious as well as the similarity between (22) and (23). Thus (23) expresses the basic ideas formulated previously in the two parts of the old seasonality criterion (3).

A more direct analogue to (17) can also be derived: From (23) we find

(24) $\sum_{\tau=0}^{s-1} z_{t-\tau}-\sum_{\tau=0}^{s-2} \frac{s-1-\tau}{s-1} w_{t-\tau}=$ constant(4). 
The impact of a perturbation in the procese (23) on the seasonal pattem

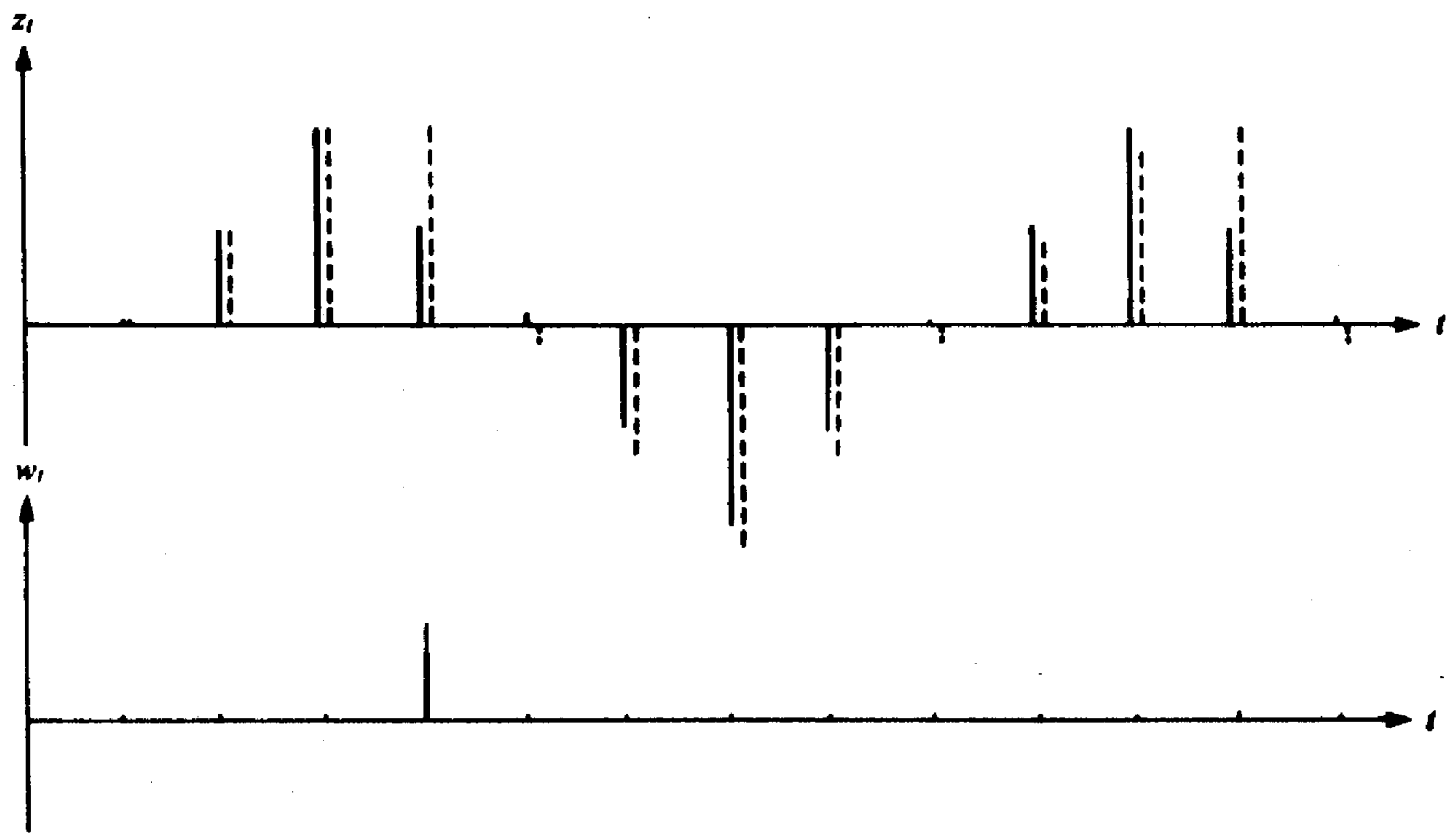

This constant can be interpreted as the sum of the undisturbed seasonal components and should hence be taken to be zero:

(25) $\sum_{t=0}^{s-1} z_{t-\tau}=\sum_{t=0}^{s-2} \frac{s-1-\tau}{s-1} w_{t-\tau}$

This can be written in matrix form as

(26) $R z=Z w$

where $R$ is as defined before in (7) and $Z$ is

(27)

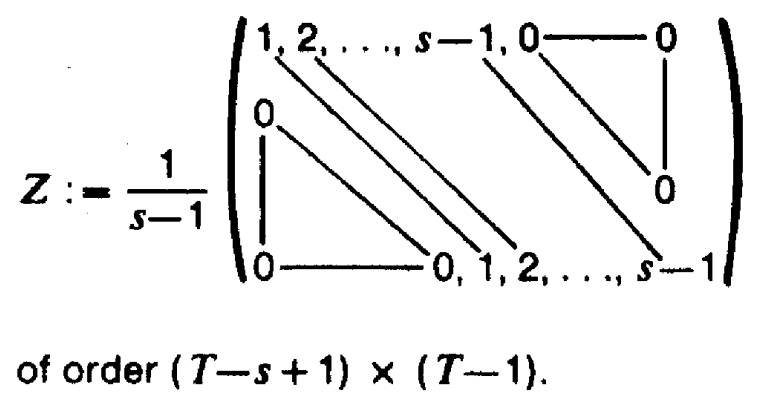

22 
The new seasonality criterion requires the minimization of the inner product $w^{\prime} w$. As a first step, $w^{\prime} w$ can be minimized for given $z$ under the constraint (26). This leads to the Lagrange condition

$$
w=Z^{\prime} \lambda
$$

where $\lambda$ is the vector of associated Lagrange multipliers. Using this together with (26) implies

(29) $\quad R z=Z Z \cdot \lambda$.

Since $Z$ has full rank, the inverse $\left(Z Z^{\prime}\right)^{-1}$ exists and we can write (28) as

$$
w=Z^{\prime}\left(Z Z^{\prime}\right)^{-1} R z
$$

The new seasonality criterion is given by the sum of squares $\gamma \cdot w^{\prime} w$ of these perturbations. It is obtained by replacing $S$ by $Z^{\prime}\left(Z Z^{\prime}\right)^{-1} R$ in (3):

$$
g^{*}(z)=\gamma z^{\prime} R^{\prime}\left(Z Z^{\prime}\right)^{-1} R z \quad \gamma>0
$$

This is the new seasonality criterion measuring the irregularity of the seasonal pattern.

\section{The decomposition theorem}

The following proposition will be proved now:

Theorem: For any given time series $x$, the minimum of

(32) $V=\alpha y^{\prime} P^{\prime} P y+\gamma z^{\prime} R^{\prime}\left(Z Z^{\prime}\right)^{-1} R z+(x-y-z)^{\prime}(x-y-z)$

with respect to $y$ and $z$ is uniquely defined by the equation

$$
H^{*}\left(\begin{array}{l}
y \\
z
\end{array}\right)-\left(\begin{array}{l}
x \\
x
\end{array}\right)
$$

where

(34) $H^{*}:=\left(\begin{array}{cc}\alpha P^{\prime} P+I, & I \\ I & , \gamma R^{\prime}\left(Z Z^{\prime}\right)^{-1} R+I\end{array}\right)$ 
Proof: We have

(35) $\frac{1}{2} \frac{\delta V}{\delta\left(\begin{array}{l}y \\ z\end{array}\right)}=H^{*}\left(\begin{array}{l}y \\ z\end{array}\right)-\left(\begin{array}{l}x \\ x\end{array}\right)$

(36) $\frac{1}{2} \frac{\delta^{8} V}{\delta\left(\begin{array}{l}y \\ z\end{array}\right)^{2}}=H^{*}$.

Since we can write

(37) $H^{*}=K^{*} K$

where

(38) $K^{\prime}:=\left(\begin{array}{cccc}\sqrt{\alpha} P^{\prime}, \sqrt{1 / 2} I, & 0 & , \sqrt{1 / 2} I \\ 0 & , \sqrt{1 / 2} I, \sqrt{\gamma} R^{\prime}\left(Z Z^{\prime}\right)^{-1} Z, \sqrt{1 / 2} I\end{array}\right)$

the matrix $H^{*}$ is nonnegative definite. Hence (33) is a necessary and sufficient condition for a minimum. In order to prove existence and uniqueness of the solution it is sufficient to prove that $H^{*}$ is nonsingular.

We have

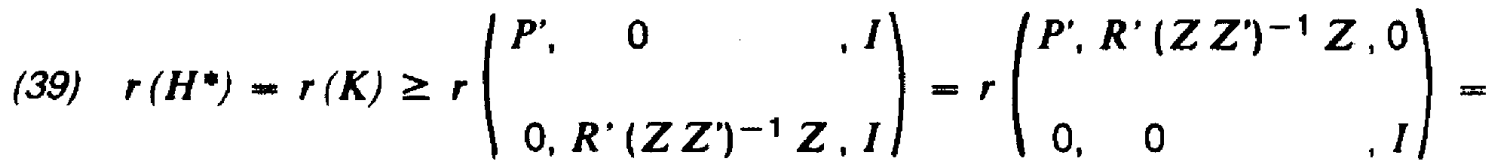

$$
\begin{aligned}
& =T+r\left(\begin{array}{c}
P \\
Z^{\prime}\left(Z Z^{\prime}\right)^{-1}
\end{array}\right)
\end{aligned}
$$

Since 
(40)

$$
r\left(\begin{array}{l}
P \\
Z^{\prime}\left(Z Z^{\prime}\right)^{-1} R
\end{array}\right) \geq r\left(\left(\begin{array}{ll}
I & 0 \\
0 & Z^{\prime}
\end{array}\right)\left(\begin{array}{c}
P \\
Z^{\prime}\left(Z Z^{\prime}\right)^{-1} R
\end{array}\right)=r\left(\begin{array}{l}
P \\
R
\end{array}\right)-T\right.
$$

where the last equality has been proved in Schlicht (1981), the proof is completed.

Hence the new seasonality criterion $g^{*}(z)$ leads to a unique decomposition of the time series into trend, seasonal component, and irregular component.

The associated regression problem would read - analogously to (19), (20):

(41) minimize $u^{\prime} u+\alpha v^{\prime} v+\gamma \hat{w}^{\prime}\left(Z Z^{\prime}\right)^{-1} \hat{w}$

with respect to $y$ and $z$ subject to

(42)

$$
\left(\begin{array}{l}
x \\
0 \\
0
\end{array}\right)=\left(\begin{array}{rr}
I, & I \\
-P, & 0 \\
0, & -R
\end{array}\right)\left(\begin{array}{l}
y \\
z
\end{array}\right)+\left(\begin{array}{c}
u \\
v \\
\hat{w}
\end{array}\right)
$$

This would lead to the same solution. The weighting matrices applied to the perturbations $u, v$, and $\hat{w}$ are $I, \alpha I$, and $\gamma\left(Z Z^{\prime}\right)^{-1}$, respectively(5). Schlicht (1983) gives a stochastic motivation for that.

\section{Concluding comments}

Under the "perturbations" point of view, the seasonality criterion proposed here reflects the two basic ideas underlying the two parts of the old seasonality criterion in one closed expression while avoiding the shortcomings of each of them.

There remains much freedom, of course, concerning the choice of the weights $\alpha$ and $\gamma$. Their meaning is quite obvious, however: They reflect the supposed variability of the perturbations. The trend will be smooth and the seasonal pattern will be stable when the shocks $v$ and $w$ are assumed to be small. This amounts to choosing large values for $\alpha$ and $\gamma$. In the descriptive framework the effect of alternative weights might be investigated by an evaluation of the sums of squared ex-post prediction errors.

The decomposition problem can be formulated stochastically, however, by interpreting $u$, $v$, and $w$ as white noise with covariance matrices $\sigma_{u l}^{2} I, \sigma_{v}^{2} I$, and $\sigma_{k}^{2} I$. This would lead to a regression problem formally similar to (41) and (42), but involving an initial value problem. (See the approaches by Akaike, 1980, Pauly, 1982. Schlicht, 1983, solves the problem without recourse to initial values, however.) 
Estimates for $\sigma_{u}^{2}, \sigma_{v}^{2}$, and $\sigma_{w}^{2}$ could be obtained, and the weights can be taken as $\alpha=\sigma_{u}^{2} / \sigma_{v}^{2}$ and $\gamma=\sigma_{u}^{2} / \sigma_{w}^{2}$. However, an exact treatment is beyond the scope of this paper.

Two descriptive features of the method proposed here are perhaps worth mentioning. Denote by $y(x)$ and $z(x)$ the trend and the seasonal components belonging to a given time series $x, i$. the solution to (33). These functions are linear:

$$
\text { (43) } \begin{aligned}
& y\left(x^{1}+x^{2}\right)=y\left(x^{1}\right)+y\left(x^{2}\right) \\
& z\left(x^{1}+x^{2}\right)=z\left(x^{1}\right)+z\left(x^{2}\right) \\
& \text { for all } x^{1}, x^{2} \in \mathrm{R}^{T}
\end{aligned}
$$

This is a reasonable descriptive property which gives a justification for using quadratic criteria.

Furthermore the method is what Burman (1979) called "weakly symmetric": Define the matrix

(44)

$$
J=\left(\begin{array}{lll}
0 & -0 & 1 \\
1 / 1 & 0 \\
0 & 1 & 1 \\
1 & 0 & 0
\end{array}\right)
$$

This matrix, applied to $x$, reverses the time sequence, i. e.

$$
J \cdot\left(\begin{array}{c}
x_{1} \\
\cdot \\
x_{T}
\end{array}\right)=\left(\begin{array}{c}
x_{T} \\
\cdot \\
\cdot \\
x_{1}
\end{array}\right)
$$

It is immediate that a seasonal adjustment of the reversed series gives the same result as the reverse of the seasonally adjusted original series $(6)$ :

$$
\begin{aligned}
& y(J x)=J y(x) \\
& z(J x)=J z(x)
\end{aligned}
$$

This seems to be a sensible feature of a descriptive method and it illustrates that a causal model, where perturbations affect only the future, is not inconsistent with the use of weakly symmetric filters. Hence this implies an objection against de Vos' (1976) argument in favour of onesided filters. With regard to empirical applications of the method, see Astier - Duhamel (1982). 


\section{References}

Akaike, H., "Seasonal Adjustment by a Bayesian Modeling", Journal of Time Series Analysis, 1980, 1(1), pp. 1-13.

Astier, R., Duhamel, C., Désaisonnalisation d'une série chronologique par minimisation des perturbations, Centre d'études sur la modèlisation et la statistique, Université de Paris-Sud, 1982, mimeo.

Burman, J. P., "Seasonal Adjustment - A Survey", in Makridakis, S., Wheelwright, S. C. (Eds.), Forecasting, Amsterdam, North Holland, 1979, pp. 45-57.

Kitagawa, K., "A Nonstationary Time Series Model and its Fitting by a Recursive Filter", Journal of Time Series Analysis, 1981, 2(2), pp. 103-116.

Leser, C. E. V., "Estimation of Quasi-Linear Trend and Seasonal Variation", Journal of the American Statistical Association, 1963, 58, pp. 1033-1043.

Pauly, A., "Zerlegung und Analyse ökonomischer Zeitreihen", Statistische Hefte, 1982, 23(4), pp. 291-303.

Schlicht, E., "A Seasonal Adjustment Principle and a Seasonal Adjustment Method Derived from this Principle", Journal of the American Statistical Association, 1981, 76(374), pp. 374-378.

Schlicht, E., "Seasonal Adjustment in a Stochastic Model", Statistische Hefte, 1983.

Sims, C. A., "Macroeconomics and Reality", Econometrica, 1980, 48(1), pp. 1-48.

de Vos, A., Seasonal Adjustment of Unemployment Figures: Criteria and Models, Paper presented at the Econometric Society European Meeting in Helsinki, Amsterdam, Free University, Interfaculty Actuarial Sciences and Econometrics. 1976, mimeo.

\section{Notes}

(1) A random shock interpretation - as in Leser (1963) with regard to the trend, in Akaike (1980) and in Kitagawa (1981) - would involve some conceptual problems regarding the relevance of our methodology of studying the properties of the criteria by tracing the system's response to so-called "typical shocks". This technique has been described by Sims (1980).

(2) With regard to Akaike's (1980) Bayesian formulation, similar objections can be raised. Akaike's (1980) approach differs from (19), (20) in that the rectangular matrices $P, R$, and $Q$ are augmented to square matrices. This induces an initial value problem for $y$ and $z$.

(3) The alternative formulation

(23) $z_{t}-z_{t}+w_{t}-\frac{1}{s} \sum_{s=0}^{s-1} w_{t-r}$ 
is equivalent since it can be obtained from (23) by replacing $w_{l}$ by $w_{1} \cdot \frac{s-1}{s}$.

(4) Denote the first term in (24) by $Z_{t}$ and the second term by $W_{t}$. Equation (23) can be re-written equivalently as $Z_{t}-Z_{t-1}-W_{t}-W_{t-1}$. Hence $Z_{t}-W_{1}=Z_{t-1}-W_{t-1}$ for all $t$ which implies (24). This formula states that the impact of a perturbation on $Z_{1}$ is linearily declining over time.

(5) 1. e. $\hat{w}=Z \boldsymbol{w}$.

(6) For matrices $J$ of appropriate order, the following holds true: $J=J, J J=I, J P J=P, J R J=$ $R, J Z Z^{\prime} J=Z Z^{\prime}$. Together with (33) and (34), this implies (46).

\section{Addresses:}

\section{Ekkehart Schlicht}

Darmstadt Institute of Technology

Schloß

D-61 Darmstadt

Ralf Pauly

University of Osnabrück

Luisenstraße 16

D-45 Osnabrück 